\title{
Stress materno e hospitalização infantil pré-cirúrgica'
}

\section{Maternal stress and presurgical infant hospitalization}

\author{
Luciana Esgalha CARNIER ${ }^{2}$ \\ Olga Maria Piazentin Rolim RODRIGUES ${ }^{2}$ \\ Flávia Helena Pereira PADOVANI ${ }^{3}$
}

\begin{abstract}
Resumo
O objetivo deste estudo foi a valiar o nível de stress de mães acompanhantes de crianças hospitalizadas para realização de procedimentos cirúrgicos eletivos, relacionando-o com variáveis demográficas da criança e experiência prévia no contexto. Participaram 54 mães acompanhantes de crianças hospitalizadas para realização de cirurgia eletiva. Utilizaram-se como instrumentos o Inventário de Sintomas de Stress para Adultos de Lipp e, para levantamento das variáveis da criança, um questionário elaborado para o estudo. Os resultados apontaram que 82\% da amostra apresentavam stress, prevalencendo a fase de resistência e sintomas psicológicos. Observou-se relação estatisticamente significativa entre a presença de stress materno e o fato de o filho não ter experiência anterior com cirurgia $(p=0,052)$. Por outro lado, não foram observadas diferenças estatisticamente significativas entre a presença de stress materno e a idade e gênero da criança. Conclui-se que o impacto dos procedimentos cirúrgicos acomete a criança e a família, o que deve ser levado em consideração a fim de que sejam propostas intervenções para a preparação pré-operatória.
\end{abstract}

Unitermos: Crianças hospitalizadas. Mães. Stress.

\begin{abstract}
The aim of this study was to evaluate the level of stress in mothers accompanying children hospitalized to have elective surgical procedures performed, relating it to demographic variables of the child and previous experience in this context. Fifty-four mothers accompanying children hospitalized to have elective surgery performed participated in the study. The instruments used were the Lipp Stress Symptoms Inventory for adults, and a questionnaire drawn up for the study to survey the children's variables. The results pointed out tht $82 \%$ of the sample presented stress, with the stage of resistance and psychological symptoms prevailing. A statistically significant relationship was observed between the presence of maternal stress and the fact that the child had no previous experience of surgery $(p=0.052)$. On the other hand, no statistically significant differences were observed between the presence of maternal stress and the child's age and gender. It was concluded that the impact of surgical procedures affects the child and family, which must be taken into consideration so that interventions can be proposed for pre-operative preparation.
\end{abstract}

Uniterms: Child, hospitalized. Stress. Mothers.

\section{VVV}

1 Artigo elaborado a partir da dissertação de L. E. CARNIER, intitulada "Stress e coping em crianças hospitalizadas em situação pré-cirurgica e stress do acompanhante estabelecendo relações". Universidade Estadual Paulista Júlio de Mesquita Filho, 2010.

2 Universidade Estadual Paulista Júlio de Mesquita Filho, Faculdade de Ciências. Av. Eng. Edmundo C. Coube, 14-01, 17033-360, Bauru, SP, Brasil. Correspondência para/Correspondence to: O.M.P.R. E-mail: <olgarolim@uol.com.br>.

3 Universidade Estadual Paulista Júlio de Mesquita Filho, Faculdade de Medicina de Botucatu, Departamento de Neurologia, Psicologia e Psiquiatria. Botucatu, SP, Brasil. 
O conceito de stress foi formulado por Hans Selye, que, em seus trabalhos com animais de laboratório, percebeu que eles excediam sua capacidade de defesa, apresentando uma série de alterações fisiológicas quando submetidos a situações hostis (Carvalho, 2004). Em 1936, Selye definiu stress como resultado inespecífico de qualquer demanda sobre o corpo, podendo ter efeitos mentais ou somáticos; e conceituou estressor como todo agente ou demanda que evoca reação de stress, seja de natureza física, mental ou emocional (Camelo \& Angerami, 2004; Goulart Jr. \& Lipp, 2008).

Em 1956, Selye descreveu a Síndrome Geral de Adaptação (SGA), pressupondo que o processo de stress compreende três fases ou estágios (Camelo \& Angerami, 2004; Goulart Jr. \& Lipp, 2008). A primeira, denominada fase de alarme, inicia-se quando a pessoa se defronta com um estressor, havendo um desequilíbrio homeostático e, então, o organismo se prepara para "luta ou fuga". Portanto, tal reação pode ser considerada saudável e benéfica ao organismo, na medida em que o prepara para atuar em situações de urgência. De acordo com Goulart Jr. e Lipp (2008), o problema surge quando a prontidão fisiológica não é necessária ou quando é excessiva.

A fase seguinte, denominada resistência, ocorre quando a fase de alerta persiste, devido à longa duração ou à grande intensidade do estressor, e o organismo se utiliza das reservas de energia adaptativa, na tentativa de se reequilibrar. Caso a reserva de energia adaptativa seja suficiente, a pessoa se recupera e sai do processo de stress. Porém, se o estressor exigir mais esforço de adaptação do que é possível para aquele indivíduo, o organismo enfraquece e torna-se vulnerável a doenças (Camelo \& Angerami, 2004; Goulart Jr. \& Lipp, 2008).

Por fim, tem-se a fase da exaustão, ou seja, o organismo encontra-se esgotado pelo excesso de atividades e pelo alto consumo de energia gasto na tentativa de buscar o restabelecimento do equilíbrio homeostático. Tal fase ocorre quando a resistência da pessoa não foi suficiente para lidar com a fonte de stress ou se houver, concomitantemente, a ocorrência de outros estressores (Camelo \& Angerami, 2004; Goulart Jr. \&Lipp, 2008).

Lipp propôs uma fase intermediária entre as de resistência e de exaustão, que denominou fase de quase-exaustão. Ela se caracteriza por um enfraquecimento da pessoa que não está conseguindo adaptar-se ou resistir ao estressor, mas que ainda não tenha atingido a exaustão completa (Camelo \& Angerami, 2004; Goulart Jr. \& Lipp, 2008; Lipp, 2004).

Independentemente da fase, o stress pode ter fontes internas ou externas. Internas são as cognições e experiências anteriores do indivíduo, que dependem de sua personalidade e do modo como ele reage às situações do dia a dia, às experiências anteriores e a seus pensamentos. Já as fontes externas estão ligadas aos estressores provenientes do ambiente e das relações que a pessoa estabelece com eles (Lipp, 2004; Lipp, Pereira \& Sadir, 2005).

O stress pode ser considerado, portanto, um processo complexo, que envolve aspectos bioquímicos, físicos e psicológicos. Caracteriza-se por um conjunto de reações que o organismo emite quando é exposto a situações/estímulos que levam a uma quebra de sua homeostase. Cabe ressaltar a importância da interpretação que o indivíduo dá aos estímulos externos e internos, tomando-os como eventos estressores, para que seja desencadeada uma resposta de adaptação do organismo, a fim de preservar sua integridade e sua própria vida (Goulart Jr. \& Lipp, 2008; Lipp, 2004).

A identificação da presença de stress e da fase em que o indivíduo se encontra possibilita o encaminhamento da pessoa para serviços especializados, com o objetivo de eliminar os desconfortos decorrentes de sua presença, principalmente nas fases mais críticas (Lipp, 2004). Para avaliar o stress, Lipp (2002) propôs o Inventário de Sintomas de Stress para adultos de Lipp (ISSL), que permite um diagnóstico preciso da presença ou não do quadro de stress, da fase em que a pessoa se encontra e de quais os sintomas tipicamente manifestados por ela (f́́sicos ou psicológicos). Esse instrumento tem sido utilizado para identificar o stress associado à profissão, como o de professores (Goulart Jr. \& Lipp, 2008; Martins, 2007), policiais (Costa, Accioly Jr., Oliveira \& Maia, 2007) e cuidadores (Horiguchi \& Lipp, 2010). Também tem sido utilizado para avaliar a eficácia de procedimentos para o combate ao stress, como o efeito da ginástica laboral, em estudo realizado por Tanaka, Deutsch e Valim (2007), além de verificar o efeito da técnica de relaxamento progressivo em portadores de disfunções temporomandibulares (Esgalha, 2009). Outros estudos para avaliação de stress em adultos com queixas médicas 
de lesão dermatológica e cefaleia, pelo ISSL, mostraram uma prevalência das fases de resistência e quase exaustão e da sintomatologia psicológica (Galego, Moraes, Cordeiro \& Tognola, 2007; Ludgwig, Oliveira, Müller \& Gonçalves, 2008).

Dentre os fatores que podem desencadear o stress, estão as questões relacionadas a doenças, agudas e principalmente crônicas, assim como os tratamentos médicos consequentes que podem ser considerados importantes eventos estressores, não só para adultos, mas também para crianças e familiares (Chorney \& Kain, 2010; Coyne, 2006; Del Bianco Faria \& Cardoso, 2010). Doenças e tratamentos podem causar, além do stress para o paciente, o stress familiar, que acomete diferentemente os membros de uma família. Não há um conceito único de stress familiar, mas, como defende Urbano (2004), ele pode estar relacionado a aspectos do próprio ambiente, ou ser decorrente de outra fonte de stress que traga repercussões para a família. O autor enfatiza que, entre os estressores familiares (relacionamentos conflituosos, filhos com problemas, sejam eles de aprendizagem ou emocionais, pressões e exigências excessivas) está a doença de um filho. Por exemplo, Fávero e Santos (2005) e Gomes e Bosa (2004) estudaram a presença de crianças autistas e o stress familiar, levando em consideração a qualidade das interações familiares.

De outro lado, Castro e Piccinini (2002) destacam que, ao mesmo tempo em que a família é afetada pela situação de doença da criança necessitada de cuidados, tal situação pode funcionar como moderadora dos efeitos negativos da doença para a criança. Os autores salientam que o suporte familiar e as competências de cada membro da família são importantes fontes de informação para a criança e podem influenciar o modo como ela lida com a doença.

O stress de cuidadores vem sendo estudado em diferentes contextos. Iwamoto, Santos, Skare e Spelling (2008) avaliaram o stress psicológico de 40 cuidadores primários de pacientes pediátricos com Artrite Idiopática Juvenil (AIJ), uma doença sistêmica inflamatória crônica, em acompanhamento ambulatorial. Para a avaliação do impacto subjetivo dos cuidados de pacientes com doenças crônicas, os autores utilizaram o questionário Caregiver Burden Scale, em sua versão validada para o português, concluindo que não só as crianças, mas também os cuidadores, necessitam de atenção e cuidados médicos.
Ao avaliar o nível de stress de vinte cuidadores de crianças (zero a 12 anos) com leucemia, por meio do ISSL, após um ano de tratamento, Del Bianco Faria e Cardoso (2010) concluíram que metade dos cuidadores apresentava indicadores de stress, com predomínio da fase de resistência (60\%) e sintomatologia majoritariamente psicológica (90\%).

O próprio tratamento, com procedimentos invasivos e dolorosos, pode se constituir um evento estressor. Em um estudo com crianças em tratamento dentário, Cardoso, Loureiro e Nelson-Filho (2004) observaram que os indicadores de stress psicológico e medo odontológico apresentados pelas mães de crianças com altos níveis de ansiedade e necessitadas de contenção física frente ao tratamento, foram maiores do que os índices apresentados pelas mães de crianças colaborativas com o atendimento. Os indicadores de stress materno, avaliados por meio do Inventário de Sintomas de Stress para Adultos de Lipp (ISSL), foram principalmente de natureza psicológica: ansiedade, irritação, hipersensibilidade emocional, falta de autoconfiança e capacidade reduzida para lidar com as dificuldades da criança.

No contexto hospitalar, as crianças estão expostas à probabilidade de serem submetidas a procedimentos invasivos e traumáticos e, por isso, a situação de internação é considerada um fator estressor para o desenvolvimento infantil. Além disso, pelo menos dois outros eventos podem também ser considerados estressores: o tempo de permanência no hospital e a hospitalização recorrente (Carvalho, Silva, Santos \& Camargo, 2006; Moro \& Módolo, 2004).

O período pré-cirúrgico envolve uma sobre-carga emocional para toda a família, podendo desencadear ou agravar sintomas de stress e ansiedade. A ansiedade pré-operatória dos pais parece ser muito comum e causada pela separação do filho, ao observarem o stress deste quando submetido a procedimentos invasivos e dolorosos, como anestesia e cirurgia. Entre os fatores que podem estar associados à ansiedade estão: a pouca idade criança, o primeiro contato desta com uma cirurgia, ser filha(o) única(o), os pais trabalharem na área da saúde (Moro \& Módolo, 2004). Os autores ressaltam tais variáveis, além das preocupações resultantes da própria cirurgia, como ser ela exitosa, garantindo não só a sobrevida da criança, mas a melhora dos sintomas. 
Ao avaliar 134 pais de crianças com idade entre 2 e 10 anos, no momento pré-operatório e após uma semana da operação, Bevan et al. (1990) evidenciaram que a ansiedade dos pais no dia da cirurgia do filho está altamente relacionada à ansiedade da criança. Dado semelhante foi encontrado por Kain, Mayes, O'Connor e Cicchetti (1996), que entrevistaram 163 pais de crianças com idade semelhante, em situação pré-cirúrgica.

Mesmo cirurgias eletivas, que não necessariamente envolvem incômodos imediatamente anteriores ou risco iminente de morte para a criança, por serem previamente agendadas, também podem resultar em ansiedade dos pais (Broering \& Crepaldi, 2008). Entre elas estão: postectomia, adenoidectomia, amigdalectomia, cirurgias para retirada de hérnia, entre outras. Uma característica dessas cirurgias é que, quando realizadas, a criança esteja gozando de boa saúde, ainda que tenha alguma doença crônica, como câncer. Shirley, Thompson, Kenward e Johnston (1998) avaliaram o nível de ansiedade de 100 pais de crianças agendadas para cirurgias eletivas, por meio de uma escala de autoavaliação (The Leeds scale for self-assessment of anxiety), everificaram que 42\% deles apresentavam níveis de ansiedade acima do esperado. O mesmo estudo verificou ainda que as mães apresentavam níveis mais elevados de ansiedade que os pais. Todavia, independentemente do nível de ansiedade demonstrado pelos pais, vários deles apontaram que acompanhar a criança no tratamento (68\%), estar presente durante a indução da anestesia (54\%) e receber mais informações da equipe no período pré-operatório (40\%) contribuem para a redução do stress.

Na tentativa de comparar a ansiedade de mães de crianças que realizariam cirurgia com a de mulheres que sofreriam cirurgia, MacLaren e Kain (2008) realizaram um estudo nos Estados Unidos, no qual utilizaram o Inventário de Ansiedade Traço-Estado (IDATE). Os resultados revelaram que as mães eram tão ansiosas quanto as mulheres submetidas à cirurgia de grande porte, e mais ansiosas que estas nas cirurgias de pequeno porte. Além disso, observou-se que a ansiedade materna estava relacionada principalmente com a pouca idade, tanto da mãe quanto da criança, e com o modo como a mãe lidava com a situação. Não houve relação entre ansiedade materna e sexo da criança, experiência anterior com hospitalização ou cirurgia, bem como com

318 o temperamento da criança.
Considerando o impacto dos procedimentos cirúrgicos, não só para a criança, mas para a família, alguns estudos têm focalizado a preparação para cirurgias também aos familiares, por meio de intervenções educativas e/ou psicológicas. Esses estudos verificaram benefícios advindos da inserção dos familiares na preparação para a cirurgia das crianças, como níveis mais baixos de ansiedade (Felder-Puig et al., 2003; Gorayeb et al., 2009), maior participação nos cuidados da criança, além dos benefícios para a própria criança (Felder-Puig et al., 2003).

Dessa forma observa-se que, na literatura analisada acerca do impacto da situação pré-cirúrgica sobre os cuidadores, prevalecem avaliações dos níveis de ansiedade, em detrimento da presença e sintomas de stress. Tal lacuna está também associada à avaliação dos efeitos da hospitalização, envolvendo procedimentos cirúrgicos de um filho como evento estressor, acrescido às variáveis da criança submetida a cirurgia eletiva. Tais resultados podem indicar temáticas que possibilitem subsidiar o planejamento de ações interventivas com a população infantil e seus cuidadores.

Esta pesquisa teve como objetivo avaliar o nível de stress de mães acompanhantes de crianças hospitalizadas para realização de procedimentos cirúrgicos eletivos, ou seja, cirurgias que não têm caráter de urgência ou emergência e que são agendadas previamente, relacionando-o com variáveis demográficas da criança e sua experiência prévia nesse contexto.

\section{Método}

\section{Participantes}

A amostra foi composta por 54 mães, acompanhantes de crianças hospitalizadas para realização de procedimentos cirúrgicos eletivos. Destas, 54\% eram mães de meninos e 46\% mães de meninas. Dentre as mães, 43\% acompanhavam crianças com idade de 7 anos a 8 anos e 11 meses; 19\% eram mães de crianças de 9 anos a 10 anos e 11 meses, e 39\% das mães acompanhavam crianças com idade de 11 anos a 12 anos e 11 meses, que eram, ou não, portadoras de doenças crônicas como, por exemplo, câncer. No geral as cirurgias eram de postectomia, adenoidectomia, amigdalectomia, retirada de hérnia, entre outras. A amostra 
caracterizou-se como uma amostragem de conveniência, já que as mães predominaram entre os acompanhantes presentes nas enfermarias onde a pesquisa foi realizada.

Os critérios estabelecidos para a composição de amostra dos acompanhantes foram: 1) a criança ter idade entre 7 anos e 12 anos e 11 meses; 2) a criança ter conhecimento da internação para realização de cirurgia; 3) o procedimento cirúrgico não ter caráter diagnóstico (biopsia), por ser considerado, principalmente pelos acompanhantes, como processo exploratório de diagnóstico, e não como procedimento cirúrgico, apesar de ser realizado em centro cirúrgico e com uso de anestesia; 4) a criança viver a primeira experiência cirúrgica por ocasião desta pesquisa, pois existem crianças que necessitam de internação para procedimento cirúrgico de forma recorrente. Cada internação pode ter características, impactos e sentimentos associados diferentes, porém, para garantir maior variabilidade de participantes, optou-se por entrevistar a acompanhante apenas uma vez, ainda que houvesse durante o estudo outros episódios de internação.

A pesquisa foi realizada nas enfermarias do Hospital das Clínicas da Faculdade de Medicina de Botucatu (FMB), onde as crianças se encontravam hospitalizadas para realização de cirurgias eletivas; ressalta-se que, dependendo da necessidade cirúrgica, as crianças não eram internadas, como nas especialidades de urologia, oftalmologia, otorrinolaringologia, entre outras. O projeto foi aprovado pelo Comitê de Ética em Pesquisa da Universidade Estadual Paulista (Unesp) (Processo no 1596/46/01/08), preservando os direitos dos participantes envolvidos e cumprindo todas as etapas previstas na Resolução 196 da Comissão Nacional de Ética em Pesquisa (1996).

\section{Instrumentos}

O instrumento foi elaborado pela pesquisadora especialmente para este estudo, tendo como finalidade coletar dados demográficos dos participantes. O questionário era composto por 15 questões, que incluíam: dados da criança, sua experiência anterior com hospitalização acompanhada de cirurgia, e dados da acompanhante.

Elaborado por Lipp (2002), esse instrumento tem como objetivo avaliar, em adultos, a presença de stress, a fase em que a pessoa se encontra e qual a sintomatologia mais apresentada (física ou psicológica), levando em consideração as fases de stress propostas por Selye (alerta, resistência e exaustão). Nos estudos para padronização do ISSL, Lipp percebeu que deveria haver maior reflexão acerca da fase de resistência proposta pelo modelo trifásico de Selye, uma vez que as pessoas não entrariam na fase de exaustão subitamente. Na validação do instrumento, ao se realizar a análise qualitativa, a autora observa a existência de uma diferença substancial entre as pessoas que possuíam alguns sintomas e outras com elevado número deles na fase de resistência. Dessa forma, por meio da análise de agrupamento dos itens, foram encontradas duas classes para essa fase, de modo que Lipp propôs uma fase intermediária entre a de resistência e a de exaustão, à qual denominou fase de quase exaustão. Esta se caracteriza por um enfraquecimento da pessoa, que não está conseguindo adaptar-se ou resistir ao estressor, mas que ainda não atingiu a exaustão completa (Camelo \& Angerami, 2004; Goulart Jr. \& Lipp, 2008; Lipp, 2002, 2004).

O Inventário foi validado por meio de análise de consistência interna e análise fatorial, numa amostra de 1843 pessoas com faixa etária entre 15 e 75 anos, sendo encontrado um alfa de Cronbach de 0,91 para a escala geral. Na análise fatorial, separaram-se dois eixos: um incluindo itens de natureza psicológica, e outro, de natureza física (Lipp, 2002).

O instrumento contém: um caderno de respostas para o participante, no qual constam 53 afirmações, divididas em três quadros: 15 no quadro 1 (sintomas experimentados nas últimas 24 horas), 15 no quadro 2 (sintomas apresentados na última semana) e 23 no quadro 3 (sintomas apresentados no último mês); um manual para avaliação; e um crivo para correção das respostas. O participante deve referir presença ou ausência de sintomas de acordo com a instrução apresentada pelo Inventário.

\section{Procedimento}

Considerando que são mães de crianças hospitalizadas para cirurgias eletivas, de qualquer especialidade médica, elas foram abordadas antes dos procedimentos, porém, pelo menos quatro horas após a hospitalização, tempo aqui considerado suficiente para que a família vivenciasse o ambiente hospitalar. 
O projeto foi apresentado às acompanhantes, discriminando seu objetivo e as tarefas inerentes a ele, de acordo com os princípios éticos recomendados pelo Comissão Nacional de Ética em Pesquisa (1996). No caso de aceite da participação, foi solicitado que a acompanhante assinasse o Termo de Consentimento Livre e Esclarecido. Separadamente da criança, em local com garantia de privacidade, a acompanhante respondeu ao Questionário de Levantamento de Informações, que foi lido pela pesquisadora e respondido pela acompanhante. As respostas foram computadas pela pesquisadora no próprio questionário. O Inventário de Sinto- mas de Stress para adultos de Lipp (ISSL) foi aplicado como prevê o manual.

Os dados do Questionário de Levantamento de Informações foram transferidos para uma planilha, somados e computados para posteriores comparações com os resultados obtidos no ISSL, de acordo com suas proposições técnicas. Foram realizadas descrições comparativas entre os resultados encontrados no ISSL e o gênero, a idade e a experiência prévia da criança em cirurgia. As análises estatísticas apropriadas foram conduzidas, comparando os resultados obtidos no teste e

Tabela 1. Resultados do ISSL para acompanhantes de crianças do sexo masculino e feminino. Botucatu (SP), 2008.

\begin{tabular}{|c|c|c|c|c|c|c|}
\hline \multirow{2}{*}{ Variáveis } & \multicolumn{2}{|c|}{ Meninos $(n=29)$} & \multicolumn{2}{|c|}{ Meninas $(n=25)$} & \multicolumn{2}{|c|}{ Total $=54$} \\
\hline & $\%$ & $\mathrm{n}$ & $\%$ & $\mathrm{n}$ & $\%$ & $n$ \\
\hline \multicolumn{7}{|c|}{ Presença do stress } \\
\hline Sem stress & 13 & 7 & 5,5 & 3 & 18 & 10 \\
\hline \multirow[t]{2}{*}{ Com stress } & 41 & 22 & 41 & 22 & 82 & 44 \\
\hline & \multicolumn{2}{|c|}{ Meninos $(n=22)$} & \multicolumn{2}{|c|}{ Meninas ( $n=22$ ) } & \multicolumn{2}{|c|}{ Total=44 } \\
\hline \multicolumn{7}{|l|}{ Fases do stress } \\
\hline Alerta & 2 & 1 & 0 & 0 & 2 & 1 \\
\hline Resistência & 34 & 15 & 32 & 14 & 66 & 29 \\
\hline Quase-exaustão & 14 & 6 & 14 & 6 & 28 & 12 \\
\hline Exaustão & 0 & 0 & 4 & 2 & 4 & 2 \\
\hline \multicolumn{7}{|c|}{ Sintomas do stress } \\
\hline Físico & 2 & 1 & 9 & 4 & 11 & 5 \\
\hline Psicológico & 45 & 20 & 39 & 17 & 85 & 37 \\
\hline Ambos & 2 & 1 & 2 & 1 & 4 & 2 \\
\hline
\end{tabular}

ISSL: Inventário de Sintomas de Stress para Adultos de Lipp.

Tabela 2. Inventário de Sintomas de Stress para Adultos de Lipp (ISSL) - resultados dos participantes de acordo com a variável idade da criança. Botucatu (SP), 2008.

\begin{tabular}{|c|c|c|c|c|c|c|c|}
\hline \multirow{2}{*}{ Variáveis } & \multicolumn{2}{|c|}{$7-8$ anos $(n=23)$} & \multicolumn{2}{|c|}{$9-10$ anos $(n=10)$} & \multicolumn{2}{|c|}{$11-12$ anos $(n=21)$} & \multirow{2}{*}{$p$-valor } \\
\hline & $\%$ & $\mathrm{n}$ & $\%$ & $n$ & $\%$ & $n$ & \\
\hline \multicolumn{8}{|l|}{ Presença do stress } \\
\hline Sem stress & 17,4 & 4 & 10 & 1 & 23,8 & 5 & \multirow{2}{*}{0,641} \\
\hline Com stress & 82,6 & 19 & 90 & 9 & 76,2 & 16 & \\
\hline \multicolumn{8}{|l|}{ Fases do stress } \\
\hline Alerta & 4,3 & 1 & 0 & 0 & 0,0 & 0 & \multirow{4}{*}{0,849} \\
\hline Resistência & 52,2 & 12 & 60 & 6 & 52,4 & 11 & \\
\hline Quase-exaustão & 21,8 & 5 & 20 & 2 & 23,8 & 5 & \\
\hline Exaustão & 4,3 & 1 & 10 & 1 & 0,0 & 0 & \\
\hline \multicolumn{8}{|c|}{ Sintomas do stress } \\
\hline Físico & 13,0 & 3 & 0 & 0 & 9,5 & 2 & \multirow{3}{*}{$\begin{array}{l}0,383 \\
0,372\end{array}$} \\
\hline Psicológico & 65,3 & 15 & 90 & 9 & 61,9 & 13 & \\
\hline Ambos & 4,3 & 1 & 0 & 0 & 4,8 & 1 & \\
\hline
\end{tabular}


no questionário e as variáveis fornecidas pelos acompanhantes, considerando o nível de significância de 5\% $(p \leq 0,05)$.

\section{Resultados}

Primeiramente serão apresentados os dados referentes às respostas apontadas pelos acompanhantes das crianças, obtidas por meio do ISSL. Considerando o resultado total, observou-se que $82 \%$ da amostra apresentaram stress, tendo como prevalência a fase de resistência (66\%) e sintomas psicológicos (85\%), como pode ser observado na Tabela 1. Tomando como base o sexo das crianças, não houve diferença significativa, uma vez que o número de acompanhantes de meninos que apresentaram stress foi igual ao de meninas (22), ambos majoritariamente na fase de resistência.

Tomando como base três faixas etárias das crianças da amostra, não foram observadas diferenças significativas quanto à presença, à fase e aos sintomas de stress, comparando-se as mães dos três grupos (Tabela 2). Todavia, o número de participantes de uma das faixas etárias era inferior aos demais grupos, o que pode ter prejudicado a análise.

Para a variável "experiência prévia da criança em procedimentos cirúrgicos", é possível notar, na Tabela 3, uma relação estatisticamente significativa entre a presença de stress materno e o fato de o filho não ter experiência anterior com cirurgia $(p=0,052)$. Todavia, consi- derando as fases de stress e sintomas prevalentes, não foram observadas diferenças estatisticamente significativas entre os grupos com e sem experiência cirúrgica prévia (Tabela 3).

\section{Discussão}

Os resultados obtidos no presente estudo apontaram um alto índice de stress em mães acompanhantes de crianças hospitalizadas para realização de procedimentos cirúrgicos, quando comparados aos encontrados na amostra de padronização do ISSL, na qual 64\% das mulheres apresentaram stress (Lipp, 2002). Tal índice foi maior do que os encontrados em estudos que utilizaram o mesmo instrumento de avaliação do stress, com mulheres em outras condições, como professoras do Ensino Fundamental de escolas públicas (Goulart Jr. \& Lipp, 2008; Martins, 2007), estudantes de medicina (Aguiar, A.P.G.F. Vieira, K.M.F. Vieira \& Nóbrega, 2009), policiais (Costa et al. 2007; Rossetti et al., 2008) e cuidadoras (Horiguchi \& Lipp, 2010). Todavia, estudos têm mostrado que as mulheres parecem mais suscetíveis ao stress do que os homens (lawamoto et al., 2008; Aguiar et al., 2009; Allegretti, 2006).

Ainda assim, os resultados das participantes das amostras deste estudo também estão acima do observado com estudos realizados com mães de crianças em diferentes condições, cujos filhos possuem necessidades especiais (Matsukura, Marturano, Oishi \&

Tabela 3. Resultados do ISSL para acompanhantes de crianças sem e com experiência cirúrgica prévia. Botucatu (SP), 2008.

\begin{tabular}{|c|c|c|c|c|c|}
\hline \multirow{2}{*}{ Variáveis } & \multicolumn{2}{|c|}{ Sem experiência prévia $(n=31)$} & \multicolumn{2}{|c|}{ Com experiência prévia (n=23) } & \multirow{2}{*}{ p-valor } \\
\hline & $\%$ & $\mathrm{n}$ & $\%$ & $n$ & \\
\hline \multicolumn{6}{|l|}{ Presença do stress } \\
\hline Sem stress & 9,7 & 3 & 30,4 & 7 & \multirow[b]{2}{*}{0,052} \\
\hline Com stress ${ }^{*}$ & 90,3 & 28 & 69,6 & 16 & \\
\hline \multicolumn{6}{|l|}{ Fases do stress } \\
\hline Alerta & 3,2 & 1 & 0,0 & 0 & \multirow{4}{*}{0,338} \\
\hline Resistência & 58,1 & 18 & 47,8 & 11 & \\
\hline Quase-exaustão & 25,8 & 8 & 17,4 & 4 & \\
\hline Exaustão & 3,2 & 1 & 4,3 & 1 & \\
\hline \multicolumn{6}{|c|}{ Sintomas do stress } \\
\hline Físicos & 12,9 & 4 & 4,3 & 1 & 0,988 \\
\hline Psicológicos & 77,4 & 24 & 56,5 & 13 & 0,322 \\
\hline Ambos & 0,0 & 0 & 8,7 & 2 & \\
\hline
\end{tabular}

${ }^{*} p \leq 0,05$. 
Borasche, 2007), mães de crianças autistas (Schmidt \& Bosa, 2007), cuidadores de crianças com leucemia (Del Bianco Faria \& Cardoso, 2010) e de crianças com câncer (Patiño-Fernandez et al., 2008). Resultados semelhantes foram encontrados entre as mães de crianças com altos níveis de ansiedade frente ao tratamento odontológico que necessitam de contenção física (Cardoso et al., 2004).

A hospitalização de um familiar está entre os principais estressores de adultos (Urbano, 2004). Os resultados obtidos são corroborados pelos de Broering e Crepaldi (2008), Moro e Módolo (2004), Crepaldi, Rabuske e Gabarra (2006), que colocam a hospitalização como ameaçadora e causadora de ansiedade, com impacto sobre o comportamento, inclusive dos cuidadores. A situação pode provocar reações adversas como stress, ansiedade e medo, seja pela possibilidade de se evitarem procedimentos invasivos no filho, seja pela dor e pela ânsia de êxito cirúrgico, seja ainda pela separação das crianças.

Outros achados da literatura corroboram os resultados obtidos, ainda que avaliem a ansiedade. Andrade e Gorenstein (2000) e Kaplan, Sadocke Grebb (1997) ressaltam que a ansiedade é um constructo associado ao stress, o que aponta a possibilidade de comparação dos resultados de stress com os de ansiedade. Lui e Wu (1999) concluíram que mais da metade (56\%) dos pais de crianças chinesas que passaram por alguma cirurgia apresentavam sintomas elevados de ansiedade. Shirley, et al. (1998) verificaram que $42 \%$ dos pais de crianças agendadas para cirurgias eletivas apresentavam níveis de ansiedade superiores ao esperado. MacLaren e Kain (2008) observaram que mães de crianças que seriam submetidas à cirurgia apresentavam pontuação média no instrumento que avaliava ansiedade IDATE semelhante e, até maior, do que mulheres que seriam elas próprias, submetidas a procedimentos cirúrgicos. Segundo os autores, ser acompanhante de criança que passará por uma situação de cirurgia parece ser um evento tão ansiogênico quanto passar o próprio adulto pelo procedimento cirúrgico.

Entre as mães do presente estudo que apresentaram stress, predominou a fase de resistência, bem como a sintomatologia psicológica do stress, corroborando estudos anteriores (Aguiar et al., 2009; Camelo \&

322 Angerami, 2004; Cardoso et al., 2004; Del Bianco Faria \&
Cardoso, 2010; Galego, et al., 2007; Goulart Jr. \& Lipp, 2008; Ludgwig et al., 2008; Matsukura et al., 2007) que avaliaram, a partir do mesmo instrumento ISSL, diferentes populações.

Considerando as variáveis das crianças que estavam sendo acompanhadas, não foi possível observar relação estatisticamente significativa entre o stress materno e o gênero ou idade das crianças. Com relação ao gênero, os dados encontrados corroboram a literatura, apontando não haver ligação entre ansiedade parental e/ou materna e sexo da criança (MacLaren \& Kain, 2008; Moro \& Módolo, 2004; Shirley et al., 1998). Considerando a idade das crianças, os dados são contrários aos da literatura, que aponta relação entre a ansiedade materna e a pouca idade da criança (MacLaren \& Kain, 2008; Moro \& Módolo, 2004). Todavia, nos referidos estudos, participaram mães de crianças a partir de 2 anos de idade, o que pode ter interferido nos resultados obtidos. Outro fato foi a divisão em grupos etários com número diferente de crianças, o que pode ter prejudicado a análise.

Quanto ao fato de a criança ter ou não ter experiência anterior com cirurgia, os dados obtidos apontaram a presença significativa $(p=0,052)$ de stress nas mães em primeira experiência. Ainda que com populações diferentes, resultados semelhantes foram encontrados em estudo com 100 pacientes adultos, de ambos os sexos, divididos em dois grupos, com e sem experiência prévia em cirurgia, realizado por Daian, Petroianu e Alberti (2009). Na avaliação por meio do ISSL, observouse que o fato de ter sido operado previamente reduziu a tensão pré-operatória. Segundo os autores, os pacientes que se submeteram ao ato cirúrgico pela primeira vez demonstraram maior labilidade emocional, provavelmente provocada pela iminência de vivenciar um evento até então desconhecido. Por outro lado, os pacientes com experiência prévia, embora tenham-se mostrado emocionalmente sensibilizados com a cirurgia, apresentaram menor stress pré-operatório.

Os pais têm papel fundamental tanto na prevenção como no tratamento das doenças dos filhos, o que é caracterizado pelo auxílio dado a ele para que consiga dar sentido à doença e a seu tratamento. Isso é relevante na medida em que a maioria das perturbações infantis relaciona-se com processos adaptativos a situações 
adversas (Barros, 2003). Ao acompanharem as crianças durante uma internação para procedimentos dolorosos e invasivos, como é o caso das cirurgias, os pais têm uma participação ainda mais importante, afirma a autora. Esta pode ser uma explicação para o elevado nível de stress materno quando da primeira experiência do filho com procedimento cirúrgico, pois a mãe pode não se sentir segura/capaz de exercer tal papel de maneira eficiente.

Todo esse processo descrito por Barros (2003) depende do que a autora chama de "significações individuais" dos pais a respeito do processo de doença e tratamento. É a partir dessas significações que os pais elaboram modelos ou teorias sobre a importância dos determinantes de saúde infantil, sobre a possibilidade de controlar e influenciar as atitudes promotoras ou inibidoras de saúde, determinando seu comportamento diante das prescrições médicas. Pode-se inferir que uma das possibilidades de stress no momento pré-cirúrgico esteja relacionada às significações individuais das mães com relação ao procedimento a que seus filhos serão submetidos, seja por falta de informações esclarecedoras, seja por fantasias e/ou crenças.

\section{Considerações Finais}

Este estudo propôs-se avaliar a presença de stress, fases e sintomas predominantes em mães acompanhantes de crianças hospitalizadas para procedimentos cirúrgicos, relacionados a variáveis da criança como sexo, idade e experiência anterior com cirurgia. Os dados obtidos apontaram presença de stress entre as mães acompanhantes, relacionadas especificamente com a primeira experiência de cirurgia da criança. Nessa situação, tais dados são indicativos da necessidade de atendimento diferenciado às mães acompanhantes, considerando tanto o preparo da criança quanto o apoio às mães.

Com relação às variáveis da criança, como sexo e idade, para a determinação do stress materno, os dados referentes ao sexo corroboram os achados da literatura, diferentemente dos dados relacionados à idade. Para futuros estudos, sugere-se que sejam incluídos acompanhantes de crianças de pouca idade e que se observe número igual de participantes em cada faixa etária.
Os dados obtidos, associados à experiência anterior com cirurgia, remetem à necessidade, não só do preparo da criança, mas da implementação de ações que ofereçam informações e acolhimento. Elaborar instrumentos específicos para coletar dados sobre a cirurgia, crenças e sentimentos sobre o processo pode auxiliar na tomada de decisões.

Outro dado importante é associar o tipo de cirurgia à idade da criança e ao sexo. No presente estudo não foi possível analisar essa variável, devido à diversidade de procedimentos. Todavia, são informações importantes que podem requerer ações pontuais, tendo em vista a especificidade de cada processo cirúrgico.

É possível concluir que o processo cirúrgico dos filhos gera alto nível de stress nas mães acompanhantes, que devem ser alvo de intervenções com o objetivo de evitar desdobramentos para ela ou efeitos indesejáveis sobre os cuidados dispensados ao filho. Isso é ainda mais importante se for a primeira cirurgia, e mais fácil se ela for previamente planejada, como as cirurgias eletivas.

\section{Referências}

Aguiar, S. M., Vieira, A. P. G. F., Vieira, K. M. F., \& Nóbrega, J. O. (2009). Prevalência de sintomas de estresse nos estudantes de medicina. Jornal Brasileiro de Psiquiatria, 58 (1), 34-38.

Allegretti, J. (2006). Nível de stress, fontes estressoras e estratégias de enfrentamento em mulheres. 2006. Dissertação de mestrado não-publicada, Pontifícia Universidade Católica de Campinas.

Andrade, L., \& Gorenstein, C. (2000). Aspectos gerais das escalas de avaliação de ansiedade. In C. Gorenstein, L. H. S. G. Andrade \& A. W. Zuardi (Orgs.), Escalas de avaliação clínica em psiquiatria e psicofarmacologia (pp.139-144). São Paulo: Lemos-Editorial.

Barros, L. (2003). Psicologia pediátrica: perspectiva desenvolvimentista (2a ed.). Lisboa: Climepsi Editores.

Bevan, J. C., Johnston, C., Haig, M. J., Tousignant, G., Lucy, S., Kirnon, V., et al. (1990). Preoperative parental anxiety predicts behavioural and emotional responses to induction of anaesthesia in children. Canadian Journal Anaesthesia, 37 (2), 177-182.

Broering, C. V., \& Crepaldi, M. A. (2008). Preparação psicológica para a cirurgia em pediatria: importância, técnicas e limitações. Paidéia, 18 (39), 61-72.

Camelo, S. H. H., \& Angerami, E. L. S. (2004). Sintomas de estresse nos trabalhadores atuantes em cinco núcleos da saúde da família. Revista Latino-Americana de Enfermagem, $12(1), 14-21$. 
Cardoso, C. L., Loureiro, S. R., \& Nelson-Filho, P. (2004). Pediatric dental treatment: manifestations of stress in patients, mothers and dental school students. Brazilian Oral Research, 18 (2), 150-155.

Carvalho, V. A. (2004). A questão do câncer. In F. C. Ferraz \& R. M. Volich (Orgs.), Psicossoma l: psicanálise e psicossomática (pp.177-195). São Paulo: Casa do Psicólogo.

Carvalho, L. S., Silva, C. A., Santos, A. C. O., \& Camargo, C. L. (2006). The child faring the stress before surgery. An exploratory study. Online Brazilian Journal of Nursing, 5 (3). Retrieved April 14, 2010, from <http://www.uff.br/ objnursing/index.php/nursing/rt/printerFriendly/508/ 117>.

Castro, E. K., \& Piccinini, C. A. (2002). Implicações da doença orgânica crônica na infância para as relações familiares: algumas questões teóricas. Psicologia: Reflexão e Crítica, 15 (3), 625-635.

Chorney, J. M., \& Kain, Z. N. (2010). Family-centered pediatric perioperative care. Anesthesiology, 112 (3), 751-755.

Comissão Nacional de Ética em Pesquisa. (1996). Resolução 196/1996. Recuperado em fevereiro 20, 2008, disponível em<http://www.conselho.saude.gov.br/docs/ Resolucoes/Reso196.doc>.

Costa, M., Accioly Jr., H., Oliveira, J., \& Maia, E. (2007). Estresse: diagnóstico dos policiais militares em uma cidade brasileira. Revista Panamericana de Salud Pública, 21 (4), 217-222.

Coyne, I. (2006). Children's experiences of hospitalization. Journal of Child Health Care, 10 (4), 326-336.

Crepaldi, M. A., Rabuske, M. M., \& Gabarra, L. M. (2006). Modalidades de atuação do psicólogo em psicologia pediátrica. In M. A. Crepaldi, B. M. Linhares \& G. B. Perosa (Orgs.), Temas em psicologia pediátrica (pp.13-55). São Paulo: Casa do Psicólogo.

Daian, M. R.; Petroianu, A.; Alberti, L. R. (2009). Avaliação do estresse psiquiátrico em pacientes submetidos a operações de grande porte sob anestesia geral. Jornal Brasileiro de Psiquiatria, 58 (4), 245-251.

Del Bianco Faria, A. M., \& Cardoso, C. L. (2010). Aspectos psicossociais de acompanhantes cuidadores de crianças com câncer: stress e enfrentamento. Estudos de Psicologia (Campinas), 27 (1), 13-20. doi: 10.1590/S0103-166X2010 000100002.

Esgalha, L. R. (2009). Disfunção Temporomandibular (DTM): impacto do relaxamento sobre ansiedade e estresse. Dissertação de mestrado não-publicada, Universidade Estadual Paulista Júlio de Mesquita Filho, Bauru.

Fávero, M. A. B., \& Santos, M. A. (2005). Presença de uma criança autista e o stress familiar. Psicologia: Reflexão e Crítica, 18 (3), 358-369.

Felder-Puig, R., Maksys, A., Noestlinger, C., Gadner, H., Stark, H., Pflueger, A., et al. (2003). Using a children's book to prepare children and parents for elective ENT surgery: results of a randomized clinical trial. International Journal of Pediatric Otorhinolaryngology, 67 (1), 35-41.

Galego, J. C. B., Moraes, A. M., Cordeiro, J. A., \& Tognola, W. A. 324 (2007). Cefaleia crônica diária: estresse e impacto sobre a qualidade de vida. Arquivos de Neuro-Psiquiatria, 65 (4), 1126-1129.

Gomes, V. F., \& Bosa, C. (2004). Estresse e relações familiares na perspectiva de irmãos de indivíduos com transtornos globais de desenvolvimento. Estudos de Psicologia, 9 (3), 553-561.

Gorayeb, R. P., Petean, E. B. L., Pileggi, F. O., Tazima, M. F. G. S., Vicente, Y. A. M. V., \& Gorayeb, R. (2009). Importance psychological intervention for the recovery of children submitted to elective surgery. Journal of Pediatric Surgery, 44 (7), 1390-1395.

Goulart Jr., E., \& Lipp, M. E. N. (2008). Estresse entre professoras do ensino fundamental de escolas públicas estaduais. Psicologia em Estudo, 13 (4), 847-857.

Horiguchi, A. S., \& Lipp, M. E. N. (2010). Alzheimer: stress e qualidade de vida de cuidadores informais. Psychiatry on line Brasil, 15 (3), Recuperado em maio 29, 2010, disponível em <http://www.polbr.med.br/ano10/art0310.php>.

Iwamoto, V., Santos, S. H. P., Skare, T. L., \& Spelling, P. F. (2008). Evaluation of psychological stress in primary caregivers of patients with juvenile idiopathic arthritis. Jornal de Pediatria, 84 (1), 91-94.

Kain, Z. N., Mayes, L. C., O'Connor, T. Z., \& Cicchetti, D. V. (1996). Preoperative anxiety in children: predictors and outcomes. Archives of Pediatrics Adolescent Medicine, 150 (12), 1238-1245.

Kaplan, H. I., Sadock, B. J., \& Grebb, J. A. (1997). Compêndio de Psiquiatria: ciência do comportamento e psiquiatria clínica. Porto Alegre: Artes Médicas.

Lipp, M. E. N. (2002). Inventário de sintomas de stress para adultos (ISSL) (2a ed. revisada). São Paulo: Casa do Psicólogo.

Lipp, M. E. N. (2004). Stress emocional: esboço da teoria de "temas de vida". In M. E. N. Lipp. Ostress no Brasil:pesquisas avançadas (pp.17-31). Campinas: Papirus.

Lipp, M. E. N., Pereira, M. B., \& Sadir, M. A. (2005). Crenças irracionais como fontes internas de stress emocional. Revista Brasileira de Terapias Cognitivas, 1 (1), 29-34.

Ludgwig, M. W. B., Oliveira M. S., Müller, M. C., \& Gonçalves, A. M. B. F. (2008). Localização da lesão e níveis de stress em pacientes dermatológicos. Estudos de Psicologia (Campinas), 25 (3), 343-52. doi: 10.1590/S0103-166X200 8000300003.

Lui, J. C. Z., \& Wu, K. K. (1999). Parental anxiety associated with participation in anaesthetic induction in children: questionnaire survey. Hong Kong Medical Journal, 5, 21-26.

Maclaren, J., \& Kain, Z. N. (2008). A Comparison of preoperative anxiety in female patients with mothers of children undergoing surgery. Anesthesia Analgesia, 106 (3), 810-813.

Martins, M. G. T. (2007). Sintomas de stress em professores brasileiros. Revista Lusófona de Educação, 10, 109-128. Recuperado em setembro 7, 2011, disponível em <http:// www.psicologia.pt/artigos/textos/A0336.pdf>.

Matsukura, T. S., Marturano, E. M., Oishi, J., \& Borasche, G. (2007). Estresse e suporte social em mães de crianças com necessidades especiais. Revista Brasileira de Educação Especial, 13 (3), 415-428. 
Moro, E. T., \& Módolo, N. S. P. (2004). Ansiedade, a criança e os pais. Revista Brasileira de Anestesiologia, 54 (5), 728-738.

Patiño-Fernandez, A. M., Pai, A. L. H., Alderfer, M., Hwang, W-T., Reilly, A., \& Kazak, A. E. (2008). Acute stress in parents of children newly diagnosed with cancer. Pediatric Blood \& Cancer, 50 (2), 289-292.

Rossetti, M. O., Ehlers, D. M., Guntert, I. B., Leme, I. F. A. S., Rabelo, I. S., Tosi, S. M. V. D., et al. (2008). O inventário de sintomas de stress para adultos de Lipp (ISSL) em servidores da polícia federal de São Paulo. Revista Brasileira de Terapias Cognitivas, 4 (2), 108-120.

Schmidt, C., \& Bosa, C. (2007). Estresse e autoeficácia em mães de pessoas com autismo. Arquivos Brasileiros de Psicologia, 59 (2), 179-191.
Shirley, P. J., Thompson, N., Kenward, M., \& Johnston, G. (1998). Parental anxiety before elective surgery in children. Anaesthesia, 53 (10), 956-959.

Tanaka, K., Deutsch, S., \& Valim, P. C. (2007). Efeito da ginástica laboral no stress. Revista Digital (Buenos Aires), 12 (108). Recuperado em maio 31, 2010, disponível em <http://www.efdeportes.com/efd108/efeito-da-ginasticalaboral-no-estresse.htm>.

Urbano, M. F. G. (2004). Stress e qualidade de vida dos pais de crianças com leucemia e o stress da criança. In M. E. N. Lipp (Org.), O stress no Brasil: pesquisas avançadas (pp.105-112). Campinas: Papirus.

Recebido em: 10/8/2010

Versão final reapresentada em:11/11/2011

Aprovado em: 2/12/2011 
